Journal of Scientific Perspectives

Volume 2 Issue 3, 2018, pp. 61-74

E-ISSN 2587-3008 (online version)

JOURNAL OF

SCIENTIFIC

PERSPECTIVES

url: http://www.ratingacademy.com.tr/ojs/index.php/jsp

doi: $10.26900 /$ jsp.2018342245

\title{
Generalized Reverse Derivations On Closed Lie Ideals ${ }^{1}$
}

\author{
Özge ATAY, Neşet AYDIN, and Barış ALBAYRAK \\ Çanakkale Onsekiz Mart University, Department of Mathematics, ÇANAKKALE / TURKEY \\ e-mail: ozgee_atay@hotmail.com \\ Çanakkale Onsekiz Mart University, Department of Mathematics, ÇANAKKALE / TURKEY \\ e-mail: neseta@comu.edu.tr \\ Çanakkale Onsekiz Mart University, Department of Banking and Finance, ÇANAKKALE / TURKEY \\ e-mail: balbayrak77@gmail.com
}

Received 10 June 2018; Accepted 02 July 2018

Abstract: In this study, we investigate commutavity of prime ring $R$ with generalized reverse derivations $F$ and $G$. Also, we proved that if $L$ is a square closed Lie ideal, then $L$ is contained in center $Z(R)$ under given conditions in theorems.

Keywords: Prime ring, Lie ideal, Reverse derivation, Generalized reverse derivation.

2010 AMS Subject Classification: Primary 16N60; Secondary 16U80, 16W25

\section{INTRODUCTION}

Let $R$ be a ring with center $Z(R)$. Recall that $R$ is prime if for any $x, y \in R$, $x R y=(0)$ implies $x=0$ or $y=0$. An additive mapping $d$ form $R$ into $R$ is called derivation if $d(x y)=d(x) y+x d(y)$ for all $x, y \in R$. In [3], Bresar generalized concept of derivation as the following: An additive mapping $F$ from $R$ into $R$ is called generalized derivation with associated derivation $d$ if $F(x y)=F(x) y+x d(y)$ for all $x, y \in R$. In [4], Bresar and Vukman introduced reverse derivation and in [1],

\footnotetext{
${ }^{1}$ This study is the revised version of the paper (Generalized Reverse Derivations On Lie Ideal Of Rings) presented in the "2nd International Rating Academy Congress: Hope" held in Kepez / Çanakkale on April 19-21, 2018
} 
Abuabakar and Gonzalez introduced generalized reverse derivation. Let $d$ from $R$ into $R$ be an additive mapping. If $d(x y)=d(y) x+y d(x)$ holds for all $x, y \in R$, then $d$ is called right reverse derivation. Let $F$ from $R$ into $R$ be an additive mapping. If $F(x y)=d(y) x+y F(x)$ holds for all $x, y \in R$, then $F$ is called right generalized reverse derivation with associated reverse derivation $d$.

For any $x, y \in R$ denote the notation $[x, y]$ for commutator $x y-y x$ and $x \circ y$ for anti-commutator $x y+y x$. We use the following basic identities.

$$
\begin{aligned}
& \text { - }[x y, z]=x[y, z]+[x, z] y \\
& \text { - }[x, y z]=[x, y] z+y[x, z] \\
& \text { - }(x y) \circ z=x(y \circ z)-[x, z] y=(x \circ z) y+x[y, z] \\
& \text { - } x \circ(y z)=(x \circ y) z-y[x, z]=y(x \circ z)+[x, y] z
\end{aligned}
$$

Let $L$ be an additive subgroup of $R$. $L$ is said to be a Lie ideal of $R$ if $[L, R] \subseteq R$. A Lie ideal $L$ is said to be a square closed Lie ideal if $x^{2} \in L$ for all $x \in L$.

In [5], Posner showed that two important properties of prime rings with derivation. In a prime ring $R$ with $\operatorname{char} R \neq 2$, if the iterate of two derivations is a derivation, then one of them is zero, and if $d$ is a derivation and $[a, d(a)] \in Z(R)$ for all $a \in R$, then either $R$ is commutative or $d$ is zero. After that, several authors have proved commutativity theorems for prime rings with derivation and generalized derivation. Also many researchers have generalized results to ideals and Lie ideals of ring. In [2], Al-Omary and Rehman showed that if $L$ is a square closed Lie ideal of prime ring with generalized derivation, then $L \subseteq Z(R)$ under several conditions.

In this study, we generalize previous studies on prime rings with reverse derivation. Let $R$ be a prime ring with $\operatorname{char} R \neq 2, F: R \rightarrow R$ be a nonzero right generalized reverse derivation with associated right reverse derivation $d: R \rightarrow R$ and $L$ be a nonzero square closed Lie ideal of $\mathrm{R}$ such that $d(Z(L)) \neq(0)$. We study following conditions and prove $L \subseteq Z(R)$. $(i)[F(x), x] \in Z(R)$ for all $x \in L$. (ii) $F(x) \circ x \in$ $Z(R)$ for all $x \in L$. (iii) $F(x \circ y)-[x, y] \in Z(R)$ for all $x, y \in L$. (iv) $F[x, y]-x \circ y \in$ $Z(R)$ for all $x, y \in L$. $(v)[F(x), d(y)]-[x, y] \in Z(R)$ for all $x, y \in L$. (vi) $[F(x), F(y)]-[x, y] \in Z(R)$ for all $x, y \in L$. (vii) $F(x) \circ F(y)-x \circ y \in Z(R)$ for all $x, y \in L .($ viii $)[F(x), F(y)]-x \circ y \in Z(R)$ for all $x, y \in L .(i x) F(x) \circ F(y)-[x, y] \in$ $Z(R)$ for all $x, y \in L$. $(x)[F(x), F(y)]-F[x, y] \in Z(R)$ for all $x, y \in L$. $(x i)$ $F(x) \circ F(y)-F(x \circ y) \in Z(R)$ for all $x, y \in L$. (xii) $F[x, y]-[F(x), y] \in Z(R)$ for all $x, y \in L$. (xiii) $F[x, y]+[F(x), y]-[F(x), F(y)] \in Z(R)$ for all $x, y \in L$. (xiv) $F[x, y]-F(x) \circ y-[d(y), x] \in Z(R)$ for all $x, y \in L$. 
In addition, we investigate commutative property for two nonzero right generalized reverse derivations $F, G: R \rightarrow R$ with associated right reverse derivations $d, g$ : $R \rightarrow R$ respectively. We study following conditions and prove $L \subseteq Z(R)$. (i) $[F(x), G(y)]-[x, y] \in Z(R)$ for all $x, y \in L$. $(i i)[F(x), x]-[x, G(x)] \in Z(R)$ for all $x, y \in L$. (iii) $F(x) \circ x-x \circ G(x) \in Z(R)$ for all $x, y \in L$. (iv) $F[x, y]-[y, G(x)] \in$ $Z(R)$ for all $x, y \in L$. $(v) F(x \circ y)-y \circ G(x) \in Z(R)$ for all $x, y \in L$.

\section{Preliminaries}

Well-known fact about prime rings:

Remark 2.1. Let $R$ be a prime ring. For an elements $a \in Z(R)$ and $b \in R$, if $a b \in Z(R)$, then $b \in Z(R)$ or $a=0$.

Remark 2.2. Let $R$ be a prime ring with char $R \neq 2$ and $L$ be a square closed Lie ideal of $R$. Then $2 a b \in L$ for all $a, b \in L$.

Lemma 2.3. [6, Lemma 2.6] Let $R$ be a 2 -torsion free semiprime ring and $L$ be a nonzero Lie ideal of $R$. If $L$ is a commutative Lie ideal of $R, i$. e., $[x, y]=0$ for all $x, y \in L$, then $L \subseteq Z(R)$.

Lemma 2.4. [7, Lemma 2.5] Let $R$ be a 2-torsion free semiprime ring and $L$ be a nonzero Lie ideal of $R$. Then $Z(L) \subseteq Z(R)$.

\section{RESUlts}

Lemma 3.1. Let $R$ be a prime ring with char $(R) \neq 2$ and $L$ be a nonzero square closed Lie ideal of $R$. If $[x, y] \in Z(R)$ for all $x, y \in L$, then $L \subseteq Z(R)$.

Proof. Let $[x, y] \in Z(R)$ for all $x, y \in L$. Then $[r,[x, y]]=0$ for all $x, y \in L, r \in R$. Replacing $x$ by $2 x y$, we get $0=[r,[2 x y, y]]=2[r,[x y, y]]$ and using $\operatorname{char}(R) \neq 2$, we have $[x, y][r, y]=0$. Replacing $r$ by $r s$ for any $s \in R$, we find $[x, y] r[s, y]=0$ for all $x, y \in L, r, s \in R$. Since $R$ is a prime ring, we obtain

$$
[x, y]=0 \text { or }[s, y]=0 \text { for all } x, y \in L, s \in R .
$$

If $[s, y]=0$, then $y \in Z(R)$ and satisfy condition $[x, y]=0$. So, $[x, y]=0$ for all $x, y \in L$ in both cases. From the Lemma 2.3 we get $L \subseteq Z(R)$.

Lemma 3.2. Let $R$ be a prime ring with char $(R) \neq 2$ and $L$ be a nonzero square closed Lie ideal of $R$. If $x \circ y \in Z(R)$ for all $x, y \in L$, then $L \subseteq Z(R)$. 
Proof. Let $x \circ y \in Z(R)$ for all $x, y \in L$. Then $[r, x \circ y]=0$ for all $x, y \in L, r \in R$. Replacing $x$ by $2 x y$, we get $0=[r, 2 x y \circ y]=2[r, x y \circ y]$ and using $\operatorname{char}(R) \neq 2$, we have $(x \circ y)[r, y]=0$. Replacing $r$ by $r s$ for any $s \in R$, we find $(x \circ y) r[s, y]=0$ for all $x, y \in L, r, s \in R$. Since $R$ is a prime ring, we obtain

$$
x \circ y=0 \text { or }[s, y]=0 \text { for all } x, y \in L, s \in R .
$$

Let $A=\{y \in L \mid x \circ y=0$ for all $x \in L\}$ and $B=\{y \in L \mid[s, y]=0$ for all $s \in R\}$. $A$ and $B$ are additive subgroups of $L$ whose $L=A \cup B$, but a group can not be written as a union of two proper subgroups of its and hence $L=A$ or $L=B$. If $L=A$, then $x \circ y=0$ for all $x \in L$. Replacing $y$ by $2 y z$ for any $z \in L$ and using $\operatorname{char}(R) \neq 2$, we get $[x, y] z=0$ for all $x, y, z \in L$. In this equation, replacing $z$ by $[z, r]$ for any $r \in R$ we find $[x, y][z, r]=0$ for all $x, y, z \in L, r \in R$. Again replacing $r$ by $r s$ for any $s \in R$, we get $[x, y] r[z, s]=0$ for all $x, y, z \in L, r, s \in R$. Since $R$ is a prime ring, we obtain

$$
[x, y]=0 \text { or }[z, s]=0 \text { for all } x, y, z \in L, s \in R .
$$

If $[x, y]=0$, then from the Lemma 2.3 we get $L \subseteq Z(R)$. If $[z, s]=0$, then $z \in Z(R)$ for all $z \in L$ and $L \subseteq Z(R)$. If $L=B$, then $[s, y]=0$ for all $s \in R, y \in L$. Hence, we obtain $y \in Z(R)$ for all $y \in L$ and $L \subseteq Z(R)$.

Lemma 3.3. Let $R$ be a prime ring with char $(R) \neq 2,0 \neq F: R \longrightarrow R$ be a right generalized reverse derivation with associated right reverse derivation $d$ and $L$ be a nonzero square closed Lie ideal of $R$ such that $d(Z(L)) \neq(0)$. If $[F(x), x] \in Z(R)$ for all $x, y, z \in L$, then $L \subseteq Z(R)$.

Proof. Let $[F(x), x] \in Z(R)$ for all $x, y, z \in L$. Replacing $x$ by $x+y$, we get

$$
[F(x), y]+[F(y), x] \in Z(R) \text { for all } x, y \in L
$$

Since $d(Z(L)) \neq(0)$, we choose fixed element $0 \neq z \in Z(L)$ which $d(z) \neq 0$. Also, $z, d(z) \in Z(R)$ from the Lemma 2.4. Replacing $x$ by $2 x z$ in Equation (1) and using $\operatorname{char}(R) \neq 2$, we get

$d(z)[x, y]+[d(z), y] x+z[F(x), y]+[z, y] F(x)+[F(y), x] z+x[F(y), z] \in Z(R)$

In this expression, using $z, d(z) \in Z(R)$ and Equation (1), we have

$$
d(z)[x, y] \in Z(R) \text { for all } x, y \in L
$$


Hence, using $0 \neq d(z) \in Z(R)$ and Remark 2.1, we obtain $[x, y] \in Z(R)$ for all $x, y \in L$. From the Lemma 3.1 we get $L \subseteq Z(R)$.

Lemma 3.4. Let $R$ be a prime ring with char $(R) \neq 2,0 \neq F: R \longrightarrow R$ be a right generalized reverse derivation with associated right reverse derivation $d$ and $L$ be a nonzero square closed Lie ideal of $R$ such that $d(Z(L)) \neq(0)$. If $F(x) \circ x \in Z(R)$ for all $x, y, z \in L$, then $L \subseteq Z(R)$.

Proof. Let $F(x) \circ x \in Z(R)$ for all $x, y, z \in L$. Replacing $x$ by $x+y$, we get

$$
F(x) \circ y+F(y) \circ x \in Z(R) \text { for all } x, y \in L
$$

Since $d(Z(L)) \neq(0)$, we choose fixed element $0 \neq z \in Z(L)$ which $d(z) \neq 0$. Also, $z, d(z) \in Z(R)$ from the Lemma 2.4. Replacing $x$ by $2 x z$ in Equation (2) and using $\operatorname{char}(R) \neq 2$, we get

$$
(d(z) x) \circ y+(z F(x)) \circ y+F(y) \circ(x z) \in Z(R) \text { for all } x, y \in L
$$

In this expression, using $z, d(z) \in Z(R)$ and Equation (2), we have

$$
d(z)(x \circ y) \in Z(R) \text { for all } x, y \in L
$$

Hence, using $0 \neq d(z) \in Z(R)$ and Remark 2.1, we obtain $x \circ y \in Z(R)$ for all $x, y \in L$. From the Lemma 3.2 we get $L \subseteq Z(R)$.

Theorem 3.5. Let $R$ be a prime ring with char $(R) \neq 2,0 \neq F: R \longrightarrow R$ be a right generalized reverse derivation with associated right reverse derivation $d$ and $L$ be a nonzero square closed Lie ideal of $R$ such that $d(Z(L)) \neq(0)$. If one of the following conditions is satisfy, then $L \subseteq Z(R)$.

i) $F(x \circ y)-[x, y] \in Z(R)$ for all $x, y \in L$.

ii) $F[x, y]-x \circ y \in Z(R)$ for all $x, y \in L$.

iii) $[F(x), d(y)]-[x, y] \in Z(R)$ for all $x, y \in L$.

Proof. i) By hypothesis,

$$
F(x \circ y)-[x, y] \in Z(R) \text { for all } x, y \in L .
$$

Since $d(Z(L)) \neq(0)$, we choose fixed element $0 \neq z \in Z(L)$ which $d(z) \neq 0$. Also, $z, d(z) \in Z(R)$ from the Lemma 2.4. Replacing $x$ by $2 x z$ in Equation (3) and using $\operatorname{char}(R) \neq 2$, we get

$$
F((x \circ y) z+x[z, y])-x[z, y]-[x, y] z \in Z(R) \text { for all } x, y \in L
$$


In this expression, using $z, d(z) \in Z(R)$ and Equation (3), we have

$$
d(z)(x \circ y) \in Z(R) \text { for all } x, y \in L
$$

Hence, using $0 \neq d(z) \in Z(R)$ and Remark 2.1, we obtain $x \circ y \in Z(R)$ for all $x, y \in L$. From the Lemma 3.2 we get $L \subseteq Z(R)$.

ii) By hypothesis,

$$
F[x, y]-x \circ y \in Z(R) \text { for all } x, y \in L .
$$

Since $d(Z(L)) \neq(0)$, we choose fixed element $0 \neq z \in Z(L)$ which $d(z) \neq 0$. Also, $z, d(z) \in Z(R)$ from the Lemma 2.4. Replacing $x$ by $2 x z$ in Equation (4) and using $\operatorname{char}(R) \neq 2$, we get

$$
F(x[z, y]+[x, y] z)-(x \circ y) z-x[z, y] \in Z(R) \text { for all } x, y \in L
$$

In this expression, using $z, d(z) \in Z(R)$ and Equation (4), we obtain

$$
d(z)[x, y] \in Z(R) \text { for all } x, y \in L
$$

Hence, using $0 \neq d(z) \in Z(R)$ and Remark 2.1, we have $[x, y] \in Z(R)$ for all $x, y \in L$. From the Lemma 3.1 we get $L \subseteq Z(R)$.

iii) By hypothesis,

$$
[F(x), d(y)]-[x, y] \in Z(R) \text { for all } x, y \in L .
$$

Since $d(Z(L)) \neq(0)$, we choose fixed element $0 \neq z \in Z(L)$ which $d(z) \neq 0$. Also, $z, d(z) \in Z(R)$ from the Lemma 2.4. Replacing $y$ by $2 y z$ in Equation (5) and using $\operatorname{char}(R) \neq 2$, we get

$$
[F(x), d(z) y+z d(y)]-[x, y] z-y[x, z] \in Z(R) \text { for all } x, y \in L
$$

In this expression, using $z, d(z) \in Z(R)$ and Equation (5), we get

$$
d(z)[F(x), y] \in Z(R) \text { for all } x, y \in L
$$

By using $0 \neq d(z) \in Z(R)$ and Remark 2.1, we have

$$
[F(x), y] \in Z(R) \text { for all } x, y \in L
$$

Replacing $y$ by $x$ in above expression, we obtain $[F(x), x] \in Z(R)$ for all $x, y \in L$. From the Lemma 3.3 we get $L \subseteq Z(R)$.

Theorem 3.6. Let $R$ be a prime ring with $\operatorname{char}(R) \neq 2,0 \neq F: R \longrightarrow R$ be a right generalized reverse derivation with associated right reverse derivation $d$ and 
$L$ be a nonzero square closed Lie ideal of $R$ such that $d(Z(L)) \neq(0)$. If one of the following conditions is satisfy, then $L \subseteq Z(R)$.

i) $[F(x), F(y)]-[x, y] \in Z(R)$ for all $x, y \in L$.

ii) $F(x) \circ F(y)-x \circ y \in Z(R)$ for all $x, y \in L$.

iii) $[F(x), F(y)]-x \circ y \in Z(R)$ for all $x, y \in L$.

iv) $F(x) \circ F(y)-[x, y] \in Z(R)$ for all $x, y \in L$.

Proof. i) By assumption,

$$
[F(x), F(y)]-[x, y] \in Z(R) \text { for all } x, y \in L .
$$

Since $d(Z(L)) \neq(0)$, we choose fixed element $0 \neq z \in Z(L)$ which $d(z) \neq 0$. Also, $z, d(z) \in Z(R)$ from the Lemma 2.4. Replacing $x$ by $2 x z$ in Equation (6) and using $\operatorname{char}(R) \neq 2$, we have

$$
[d(z) x+z F(x), F(y)]-x[z, y]-[x, y] z \in Z(R) \text { for all } x, y \in L
$$

In this expression, using $z, d(z) \in Z(R)$ and Equation (6), we obtain

$$
d(z)[x, F(y)] \in Z(R) \text { for all } x, y \in L
$$

By using $0 \neq d(z) \in Z(R)$ and Remark 2.1, we have

$$
[x, F(y)] \in Z(R) \text { for all } x, y \in L
$$

Replacing $y$ by $x$ in above expression, we get $[x, F(x)] \in Z(R)$ for all $x, y \in L$. From the Lemma 3.3 we get $L \subseteq Z(R)$.

ii) By assumption,

$$
F(x) \circ F(y)-x \circ y \in Z(R) \text { for all } x, y \in L .
$$

Since $d(Z(L)) \neq(0)$, we choose fixed element $0 \neq z \in Z(L)$ which $d(z) \neq 0$. Also, $z, d(z) \in Z(R)$ from the Lemma 2.4. Replacing $x$ by $2 x z$ in Equation $(7)$ and using $\operatorname{char}(R) \neq 2$, we get

$$
(d(z) x+z F(x)) \circ F(y)-(x \circ y) z-x[z, y] \in Z(R) \text { for all } x, y \in L
$$

In this expression, using $z, d(z) \in Z(R)$ and Equation (7), we obtain

$$
d(z)(x \circ F(y)) \in Z(R) \text { for all } x, y \in L
$$

By using $0 \neq d(z) \in Z(R)$ and Remark 2.1, we have

$$
x \circ F(y) \in Z(R) \text { for all } x, y \in L
$$


Replacing $y$ by $x$ in above expression, we get $x \circ F(x) \in Z(R)$ for all $x, y \in L$. From the Lemma 3.4 we get $L \subseteq Z(R)$.

iii) By assumption,

$$
[F(x), F(y)]-x \circ y \in Z(R) \text { for all } x, y \in L .
$$

Since $d(Z(L)) \neq(0)$, we choose fixed element $0 \neq z \in Z(L)$ which $d(z) \neq 0$. Also, $z, d(z) \in Z(R)$ from the Lemma 2.4. Replacing $x$ by $2 x z$ in Equation (8) and using $\operatorname{char}(R) \neq 2$, we obtain

$$
[d(z) x, F(y)]+[z F(x), F(y)]-x z \circ y \in Z(R) \text { for all } x, y \in L
$$

In this expression, using $z, d(z) \in Z(R)$ and Equation (8), we get

$$
d(z)[x, F(y)] \in Z(R) \text { for all } x, y \in L
$$

By using $0 \neq d(z) \in Z(R)$ and Remark 2.1, we have

$$
[x, F(y)] \in Z(R) \text { for all } x, y \in L
$$

Replacing $y$ by $x$ in above expression, we have $[x, F(x)] \in Z(R)$ for all $x, y \in L$. From the Lemma 3.3 we get $L \subseteq Z(R)$.

iv) By assumption,

$$
F(x) \circ F(y)-[x, y] \in Z(R) \text { for all } x, y \in L .
$$

Since $d(Z(L)) \neq(0)$, we choose fixed element $0 \neq z \in Z(L)$ which $d(z) \neq 0$. Also, $z, d(z) \in Z(R)$ from the Lemma 2.4. Replacing $x$ by $2 x z$ in Equation (9) and using $\operatorname{char}(R) \neq 2$, we obtain

$$
(d(z) x \circ F(y))+(z F(x) \circ F(y))-x[z, y]-[x, y] z \in Z(R) \text { for all } x, y \in L
$$

In this expression, using $z, d(z) \in Z(R)$ and Equation (9), we get

$$
d(z)(x \circ F(y)) \in Z(R) \text { for all } x, y \in L
$$

By using $0 \neq d(z) \in Z(R)$ and Remark 2.1, we have

$$
x \circ F(y) \in Z(R) \text { for all } x, y \in L
$$

Replacing $y$ by $x$ in above expression, we have $x \circ F(x) \in Z(R)$ for all $x, y \in L$. From the Lemma 3.4 we get $L \subseteq Z(R)$.

Theorem 3.7. Let $R$ be a prime ring with char $(R) \neq 2,0 \neq F: R \longrightarrow R$ be a right generalized reverse derivation with associated right reverse derivation $d$ and 
$L$ be a nonzero square closed Lie ideal of $R$ such that $d(Z(L)) \neq(0)$. If one of the following conditions is satisfy, then $L \subseteq Z(R)$.

i) $[F(x), F(y)]-F[x, y] \in Z(R)$ for all $x, y \in L$.

ii) $F(x) \circ F(y)-F(x \circ y) \in Z(R)$ for all $x, y \in L$.

iii) $F[x, y]-[F(x), y] \in Z(R)$ for all $x, y \in L$.

Proof. $i$ ) For all $x, y \in L$, let

$$
[F(x), F(y)]-F[x, y] \in Z(R)
$$

By hypothesis, $d(Z(L)) \neq(0)$. Then, we choose fixed element $0 \neq z \in Z(L)$ which $d(z) \neq 0$. Also, $z, d(z) \in Z(R)$ from the Lemma 2.4. Replacing $x$ by $2 x z$ in Equation (10) and using $\operatorname{char}(R) \neq 2$, we get

$$
[d(z) x, F(y)]+[z F(x), F(y)]-F([x, y] z) \in Z(R) \text { for all } x, y \in L
$$

By using the fact that $z, d(z) \in Z(R)$ and Equation (10), we get

$$
d(z)([x, F(y)]-[x, y]) \in Z(R) \text { for all } x, y \in L
$$

In this expression, using $0 \neq d(z) \in Z(R)$ and Remark 2.1, we have

$$
[x, F(y)]-[x, y] \in Z(R) \text { for all } x, y \in L
$$

Replacing $x$ by $2 d(z) y$ in above expression and using $\operatorname{char}(R) \neq 2$, we obtain

$$
d(z)[y, F(y)] \in Z(R) \text { for all } x, y \in L
$$

Again, using $0 \neq d(z) \in Z(R)$ and Remark 2.1, we get

$$
[y, F(y)] \in Z(R) \text { for all } x, y \in L
$$

From the Lemma 3.3 we obtain $L \subseteq Z(R)$.

ii) For all $x, y \in L$, let

$$
F(x) \circ F(y)-F(x \circ y) \in Z(R)
$$

By hypothesis, $d(Z(L)) \neq(0)$. Then, we choose fixed element $0 \neq z \in Z(L)$ which $d(z) \neq 0$. Also, $z, d(z) \in Z(R)$ from the Lemma 2.4. Replacing $x$ by $2 x z$ in Equation (11) and using char $(R) \neq 2$, we get

$$
(d(z) x+z F(x)) \circ F(y)-F((x \circ y) z+x[z, y]) \in Z(R) \text { for all } x, y \in L
$$


By using the fact that $z, d(z) \in Z(R)$ and Equation (11), we have

$$
d(z)(x \circ F(y)-x \circ y) \in Z(R) \text { for all } x, y \in L
$$

In this expression, using $0 \neq d(z) \in Z(R)$ and Remark 2.1, we obtain

$$
x \circ F(y)-x \circ y \in Z(R) \text { for all } x, y \in L
$$

Replacing $y$ by $2 y z$ in above expression and using $\operatorname{char}(R) \neq 2$, we get

$$
d(z)(x \circ y) \in Z(R) \text { for all } x, y \in L
$$

Again, using $0 \neq d(z) \in Z(R)$ and Remark 2.1, we obtain

$$
x \circ y \in Z(R) \text { for all } x, y \in L
$$

From the Lemma 3.2 we get $L \subseteq Z(R)$.

iii) For all $x, y \in L$, let

$$
F[x, y]-[F(x), y] \in Z(R)
$$

By hypothesis, $d(Z(L)) \neq(0)$. Then, we choose fixed element $0 \neq z \in Z(L)$ which $d(z) \neq 0$. Also, $z, d(z) \in Z(R)$ from the Lemma 2.4. Replacing $y$ by $2 y z$ in Equation (12) and using $\operatorname{char}(R) \neq 2$, we get

$$
F([x, y] z+y[x, z])-[F(x), y] z-y[F(x), z] \in Z(R) \text { for all } x, y \in L
$$

By using the fact that $z, d(z) \in Z(R)$ and Equation (12), we have

$$
d(z)[x, y] \in Z(R) \text { for all } x, y \in L
$$

In this expression, using $0 \neq d(z) \in Z(R)$ and Remark 2.1, we obtain

$$
[x, y] \in Z(R) \text { for all } x, y \in L
$$

From the Lemma 3.1 we get $L \subseteq Z(R)$.

Theorem 3.8. Let $R$ be a prime ring with char $(R) \neq 2,0 \neq F: R \longrightarrow R$ be a right generalized reverse derivation with associated right reverse derivation $d$ and $L$ be a nonzero square closed Lie ideal of $R$ such that $d(Z(L)) \neq(0)$. If one of the following conditions is satisfy, then $L \subseteq Z(R)$.

i) $F[x, y]+[F(x), y]-[F(x), F(y)] \in Z(R)$ for all $x, y \in L$.

ii) $F[x, y]-F(x) \circ y-[d(y), x] \in Z(R)$ for all $x, y \in L$. 
Proof. i) By assumption,

$$
F[x, y]+[F(x), y]-[F(x), F(y)] \in Z(R) \text { for all } x, y \in L .
$$

Since $d(Z(L)) \neq(0)$, we choose fixed element $0 \neq z \in Z(L)$ which $d(z) \neq 0$. Also, $z, d(z) \in Z(R)$ from the Lemma 2.4. Replacing $y$ by $2 y z$ in Equation (13) and using $\operatorname{char}(R) \neq 2$, we get

$$
F[x, y z]+[F(x), y z]-[F(x), F(y z)] \in Z(R) \text { for all } x, y \in L
$$

In this expression, using $z, d(z) \in Z(R)$ and Equation (13), for all $x, y \in L$ we obtain

$$
d(z)[x, y]+z F[x, y]+[F(x), y] z-d(z)[F(x), y]-z[F(x), F(y)] \in Z(R)
$$

and from this

$$
d(z)([x, y]-[F(x), y]) \in Z(R) \text { for all } x, y \in L
$$

By using $0 \neq d(z) \in Z(R)$ and Remark 2.1, we have

$$
[x, y]-[F(x), y] \in Z(R) \text { for all } x, y \in L
$$

Replacing $y$ by $2 d(z) x$ in above expression and using $z, d(z) \in Z(R)$ and $\operatorname{char}(R) \neq$ 2, we have

$$
d(z)[F(x), x] \in Z(R) \text { for all } x, y \in L
$$

Again, using $0 \neq d(z) \in Z(R)$ and Remark 2.1, we get

$$
[F(x), x] \in Z(R) \text { for all } x, y \in L
$$

From the Lemma 3.3 we get $L \subseteq Z(R)$.

ii) By assumption,

$$
F[x, y]-F(x) \circ y-[d(y), x] \in Z(R) \text { for all } x, y \in L .
$$

Since $d(Z(L)) \neq(0)$, we choose fixed element $0 \neq z \in Z(L)$ which $d(z) \neq 0$. Also, $z, d(z) \in Z(R)$ from the Lemma 2.4. Replacing $y$ by $2 y z$ in Equation (14) and using $\operatorname{char}(R) \neq 2$, we have

$$
F[x, y z]-F(x) \circ y z-[d(y z), x] \in Z(R) \text { for all } x, y \in L
$$

In this expression, using $z, d(z) \in Z(R)$ and Equation (13), for all $x, y \in L$ we get

$$
d(z)[x, y]+z F[x, y]-(F(x) \circ y) z-d(z)[y, x]-z[d(y), x] \in Z(R)
$$


and from this

$$
2 d(z)[x, y] \in Z(R) \text { for all } x, y \in L
$$

By using $\operatorname{char}(R) \neq 2,0 \neq d(z) \in Z(R)$ and Remark 2.1, we obtain

$$
[x, y] \in Z(R) \text { for all } x, y \in L
$$

From the Lemma 3.1 we get $L \subseteq Z(R)$.

Theorem 3.9. Let $R$ be a prime ring with char $(R) \neq 2,0 \neq F, G: R \longrightarrow R$ are right generalized reverse derivations with associated right reverse derivation $d$ and $g$ respectively, $L$ be a nonzero square closed Lie ideal of $R$ such that $d(Z(L)) \neq(0)$ and $g(Z(L)) \neq(0)$. If one of the following conditions is satisfy, then $L \subseteq Z(R)$.

i) $[F(x), G(y)]-[x, y] \in Z(R)$ for all $x, y \in L$.

ii) $F[x, y]-[y, G(x)] \in Z(R)$ for all $x, y \in L$.

iii) $F(x \circ y)-y \circ G(x) \in Z(R)$ for all $x, y \in L$.

Proof. i) For all $x, y \in L$, let

$$
[F(x), G(y)]-[x, y] \in Z(R)
$$

By hypothesis, $d(Z(L)) \neq(0)$. Then, we choose fixed element $0 \neq z \in Z(L)$ which $d(z) \neq 0$. Also, $z, d(z) \in Z(R)$ from the Lemma 2.4. Replacing $x$ by $2 x z$ in Equation (15) and using $\operatorname{char}(R) \neq 2$, we get

$$
[d(z) x, G(y)]+[z F(x), G(y)]-x[z, y]-[x, y] z \in Z(R) \text { for all } x, y \in L
$$

By using the fact that $z, d(z) \in Z(R)$ and Equation (15), we obtain

$$
d(z)[x, G(y)] \in Z(R) \text { for all } x, y \in L
$$

In this expression, using $0 \neq d(z) \in Z(R)$ and Remark 2.1, we have

$$
[x, G(y)] \in Z(R) \text { for all } x, y \in L
$$

Replacing $y$ by $x$ in above expression, we have $[x, G(x)] \in Z(R)$ for all $x, y \in L$. From the Lemma 3.3 we get $L \subseteq Z(R)$.

ii) For all $x, y \in L$, let

$$
F[x, y]-[y, G(x)] \in Z(R)
$$

By hypothesis, $d(Z(L)) \neq(0)$. Then, we choose fixed element $0 \neq z \in Z(L)$ which $d(z) \neq 0$. Also, $z, d(z) \in Z(R)$ from the Lemma 2.4. Replacing $y$ by $2 y z$ in Equation 
(16) and using $\operatorname{char}(R) \neq 2$, we get

$$
F([x, y] z+y[x, z])-y[z, G(x)]-[y, G(x)] z \in Z(R) \text { for all } x, y \in L
$$

By using the fact that $z, d(z) \in Z(R)$ and Equation (16), we get

$$
d(z)[x, y] \in Z(R) \text { for all } x, y \in L
$$

In this expression, using $0 \neq d(z) \in Z(R)$ and Remark 2.1, we have

$$
[x, y] \in Z(R) \text { for all } x, y \in L
$$

From the Lemma 3.1 we obtain $L \subseteq Z(R)$.

iii) For all $x, y \in L$, let

$$
F(x \circ y)-y \circ G(x) \in Z(R)
$$

By hypothesis, $d(Z(L)) \neq(0)$. Then, we choose fixed element $0 \neq z \in Z(L)$ which $d(z) \neq 0$. Also, $z, d(z) \in Z(R)$ from the Lemma 2.4. Replacing $y$ by $2 y z$ in Equation (17) and using char $(R) \neq 2$, we get

$$
F((x \circ y) z-y[x, z])-(y \circ G(x)) z-y[z, G(x)] \in Z(R) \text { for all } x, y \in L
$$

By using the fact that $z, d(z) \in Z(R)$ and Equation (17), we obtain

$$
d(z)(x \circ y) \in Z(R) \text { for all } x, y \in L
$$

In this expression, using $0 \neq d(z) \in Z(R)$ and Remark 2.1, we have

$$
x \circ y \in Z(R) \text { for all } x, y \in L
$$

From the Lemma 3.2 we get $L \subseteq Z(R)$.

Example 3.10. Let $R=\left\{\left(\begin{array}{ll}x & y \\ 0 & x\end{array}\right) \mid x, y \in \mathbb{Z}\right\}$ and $L=\left\{\left(\begin{array}{ll}0 & a \\ 0 & 0\end{array}\right) \mid a \in \mathbb{Z}\right\}$, where $\mathbb{Z}$ is the set of all integers. We define the mappings $F, d: R \rightarrow R$ as following:

$$
F\left(\begin{array}{ll}
x & y \\
0 & x
\end{array}\right)=\left(\begin{array}{cc}
-x & 0 \\
0 & -x
\end{array}\right), d\left(\begin{array}{ll}
x & y \\
0 & x
\end{array}\right)=\left(\begin{array}{ll}
0 & y \\
0 & 0
\end{array}\right)
$$

It is easy to show that, $L$ is square closed Lie ideal of ring $R$, $d$ is right reverse derivation and $F$ is right generalized reverse derivation with associated d. Moreover, since $\left(\begin{array}{ll}0 & a \\ 0 & 0\end{array}\right) \in Z(L)$ and $d\left(\begin{array}{ll}0 & a \\ 0 & 0\end{array}\right)=\left(\begin{array}{ll}0 & a \\ 0 & 0\end{array}\right) \neq\left(\begin{array}{ll}0 & 0 \\ 0 & 0\end{array}\right)$ for any $0 \neq a \in \mathbb{Z}$, condition $d(Z(L)) \neq(0)$ is satisfied. 


\section{REFERENCES}

[1] ABUABAKAR A., GONZALEZ S.,2015 Generalized Reverse Derivations on Semiprime Rings, Siberian Math. Journal,56(2),199-205.

[2] AL-OMARY R. M., REHMAN N., 2016 Lie Ideals and Centralizeing Mappings with Generalized Derivations, Journal of Scientific Research and Reports, 11(3), 1-8.

[3] BRESAR M., 1991, On the Distance of the Composition of Two Derivations to the Generalized Derivations, Glaskow Math. J., 33, 89-93.

[4] BRESAR M., VUKMAN J., 1989, On some Additive Mappings in Rings with Involution, Aequation Math., 38, 178-185.

[5] POSNER E., 1957, Derivations in Prime Rings, Proc. Amer. Marh. Soc., 8, 1093-1100.

[6] REHMAN N., 2002, On commutativity of Rings with Generalized Derivations, Math. J. Okayama Univ., 44, 43-49.

[7] REHMAN N, HANGAN, M. and AL-OMARY R. M., 2014, Centralizing Mappings, Morita Context and Generalized $(\alpha, \beta)$-derivations, J. Taibah Univ. Sci., 8(4), 370-374. 